\title{
MS09-04 | ARCIMBOLDO TOWARDS LOW RESOLUTION: RECENT UPDATES
}

Borges, Rafael; Millán Nebot, Claudia Lucía (Institut de Biologia Molecular de Barcelona, Barcelona, ESP); Medina, Ana; Soler, Nicolas; Uson, Isabel (ICREA, Catalan Institution for Research and Advanced Studies, Barcelona, ESP)

In high resolution macromolecular crystallography, the use of small fragments in phasing is currently well established. A small fraction of a structure, if highly accurate, may render phases good enough to allow density modification and autotracing algorithms to complete the structure. The method implemented in ARCIMBOLDO [1] relies on the placement with PHASER [2] of ubiquitous fragments, such as $\alpha$-helices or libraries of $\beta$-sheets, followed by density modification and autotracing with SHELXE. The same principle, whereby a very incomplete but highly accurate structure, when subject to density modification reveals new elements in the structure holds at resolutions down to 3-4Å. Nevertheless, phasing becomes very challenging. The pattern recognition-based interpretation of an electron density into a model with its refinement implemented in ARP/wARP has been efficiently dealing with model completion in this range of resolution [3]. In ARCIMBOLDO, further development in ARCIMBOLDO_LITE with coil coiled mode, in ARCIMBOLDO_SHREDDER and integrating anomalous signal allowed also to address this scenario [4]. With SEQUENCE_SLIDER, impossibility to expand is overcome by improving the polyalanine chains by assembling and refining the most probable side chain conformations. Sequence hypotheses may be generated on a secondary structure basis or using the optimum alignment between a remote homologous structure and target sequences.

[1] Sammito, M.D. et al. (2016). Acta Crystallogr. Sect. Found. Adv. 72, s23.

[2] McCoy, A.J. et al., (2007). J. Appl. Cryst. 40, 658-674.

[3] Langer, G. et al. (2008). Nat. Protoc. 3, 1171-1179.

[4] Ferrero, D. et al. (2019). PLOS Pathogens (accepted). 